\title{
Increased air pollution exposure among the Chinese population during the national quarantine
} in 2020

\author{
Huizhong Shen ${ }^{1,2}$, Guofeng Shen ${ }^{2}{ }^{凶}$, Yilin Chen ${ }^{1}$, Armistead G. Russell', Yongtao Hu', \\ Xiaoli Duan $\mathbb{D}^{3}$, Wenjun Meng $\mathbb{D}^{2}$, Yang Xu $\mathbb{D}^{2}$, Xiao Yun $\mathbb{1}^{2}$, Baolei Lyu ${ }^{4}$, Shunliu Zhao ${ }^{5}$, \\ Amir Hakami ${ }^{5}$, Jianping Guo $\mathbb{1}^{6}$, Shu Tao $\mathbb{D}^{2}$ and Kirk R. Smith ${ }^{7,8}$
}

The COVID-19 quarantine in China is thought to have reduced ambient air pollution. The overall exposure of the population also depends, however, on indoor air quality and human mobility and activities. Here, by integrating real-time mobility data and a questionnaire survey on time-activity patterns during the pandemic, we show that despite a decrease in ambient $\mathbf{P M}_{2.5}$ during the quarantine, the total population-weighted exposure to $\mathbf{P M}_{2.5}$ considering both indoor and outdoor environments increased by $5.7 \mathrm{\mu g} \mathrm{m}^{-3}\left(95 \%\right.$ confidence interval, $\left.1.2-11.0 \mu \mathrm{g} \mathrm{m}^{-3}\right)$. The increase in population-weighted exposure was mainly driven by a nationwide urban-to-rural population migration before the Spring Festival coupled with the freezing of the migration backward due to the quarantine, which increased household energy consumption and the fraction of people exposed to rural household air pollution indoors. Our analysis reveals an increased inequality of air pollution exposure during the quarantine and highlights the importance of household air pollution for population health in China.

$\mathrm{D}$ ue to the outbreak of COVID-19, China activated the First Level Public Health Emergency Response (here called a quarantine), which required local governments to carry out strict restrictions on travel ${ }^{1,2}$. The entire country was under this quarantine, which lasted for one month and was arguably unprecedented regarding its spatial coverage, duration, strictness and effectiveness for preventing the spread of COVID-19 (ref. ${ }^{2}$ ). There was an observed improvement in ambient air quality during the quarantine, probably due to the limited industrial and transportation activities coupled with favourable meteorological conditions $^{3-8}$. Some expected that the air quality improvement may have reduced the exposure of the population to air pollutants, such as $\mathrm{NO}_{2}$ (refs. ${ }^{6,7,9}$ ). If coupled with reductions in the ambient levels of fine particulate matter with a diameter $<2.5 \mu \mathrm{m}\left(\mathrm{PM}_{2.5}\right)$, for which the best information is available on health impacts, the quarantine may have yielded an inadvertent health benefit during the COVID19 pandemic. How actual population exposure changed, however, depends not only on the ambient air quality but also on the air quality indoors, and the mobility and daily activity patterns of individuals, such as the time spent in different locations $\mathbf{s}^{10-13}$.

The quarantine triggered by the outbreak started on 25 January 2020 , which coincided with the start of the 2020 Spring Festival. Just before the start, reportedly 125 million migrant workers had moved from urban to rural areas to reunite with their families ${ }^{14}$. Normally, they would have returned at most one month after the start of the Festival ${ }^{15}$. The nationwide returning-to-work tide, however, was frozen by travel restrictions under the quarantine ${ }^{16,17}$. Thus, an extra $9 \%$ of the Chinese population were kept in rural areas longer because of the COVID-19 outbreak, areas where household air pollution (HAP) is more severe due to the prevalent use of solid fuels (coal and biomass) for cooking ${ }^{13,18}$. Also, during that season, there was still substantial space heating in households over much of the country, which is even more likely to be done with solid fuels than is cooking ${ }^{19}$.

The question we ask is how the overall $\mathrm{PM}_{2.5}$ exposure of the Chinese population changed during the COVID-19 quarantine, taking into account the changes in indoor and outdoor concentrations, time spent indoors and outdoors, and large-scale migration patterns. Such an assessment is of interest because of the health impacts of short-term exposure to $\mathrm{PM}_{2.5}$ (refs. ${ }^{20-22}$ ) and the reported associations between $\mathrm{PM}_{2.5}$ and the spread and severity of the COVID-19 infection ${ }^{23,24}$. Here, we use real-time migration data, during-pandemic activity survey data, national census data, advanced air quality modelling techniques, and an indoor exposure model to track the dynamic changes in the population exposure to $\mathrm{PM}_{2.5}$ across China before and during the nationwide quarantine (Methods).

\section{Results}

Overall change in $\mathrm{PM}_{2.5}$ exposure before and after the COVID19 quarantine. We focus our comparison on two periods: P1, one month preceding the Spring Festival spanning from 25 December 2019 to 24 January 2020; and P2, one month following the start of the Spring Festival, that is, the quarantine period, spanning from 25 January 2020 to 25 February 2020 (Fig. 1). Using surveys on time-activity patterns of the Chinese population both in normal

\footnotetext{
'School of Civil \& Environmental Engineering, Georgia Institute of Technology, Atlanta, GA, USA. ${ }^{2}$ College of Urban and Environmental Sciences, Laboratory for Earth Surface Processes, Sino-French Institute for Earth System Science, Peking University, Beijing, China. ${ }^{3}$ School of Energy and Environmental Engineering, University of Science and Technology Beijing, Beijing, China. ${ }^{4}$ Huayun Sounding Meteorology Technology Corporation, Beijing, China. ${ }^{5}$ Department of Civil and Environmental Engineering, Carleton University, Ottawa, Ontario, Canada. ${ }^{6}$ The State Key Laboratory of Severe Weather, Chinese Academy of Meteorological Sciences, Beijing, China. ${ }^{7}$ Environmental Health Sciences, School of Public Health, University of California, Berkeley, CA, USA. ${ }^{8}$ Deceased: Kirk R. Smith. ${ }^{凶}$-mail: gfshen12@pku.edu.cn
} 


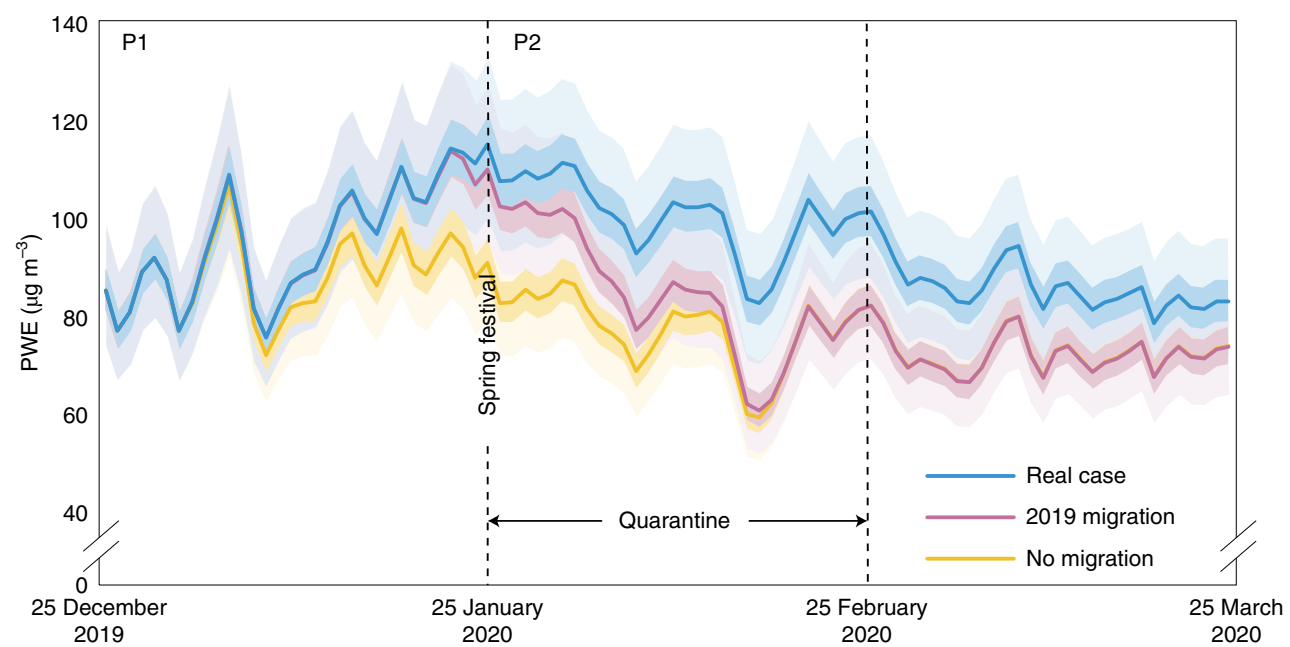

Fig. 1 | Daily trends of PWE in the real case and under different counterfactual scenarios during the study period. The dark- and light-shaded areas represent the interquartile ranges and the $95 \%$ Cls of the time series, respectively. Compared to the real case, the ' 2019 migration' scenario assumes that there was no COVID-19 outbreak such that the migration followed the pattern of the 2019 Spring Festival (instead of 2020) and the time spent indoors was not affected by the quarantine. The 'no migration' scenario assumes no COVID-19 outbreak and no Spring Festival migration. Weather conditions and ambient $\mathrm{PM}_{2.5}$ levels in the two counterfactual scenarios remain the same as in the real case. The difference between the real case and the 2019 migration scenario reflects the impacts of the quarantine-induced freezing of the migration and the change in time spent indoors on PWE. The difference between the '2019 migration' and 'no migration' scenarios reflects the impact of the Spring Festival migration on PWE in normal years. Our uncertainty analysis shows that despite the overlapping of the PWE uncertainty ranges between the real and counterfactual cases, the effects of migration and time-activity patterns on PWE are significant (Supplementary Methods 3 and 4).

days and during the quarantine (Supplementary Table 1), population time use is parsed between indoors and outdoors (Methods). Data fusion using an ensemble deep learning method to integrate the ground-level measurements of the national monitoring network with the outputs of a chemical transport model ${ }^{25}$ (Methods) shows a decrease of $16.6 \mu \mathrm{g} \mathrm{m}^{-3}\left(15.3-17.9 \mu \mathrm{g} \mathrm{m}^{-3}\right.$, uncertainty is expressed as $95 \%$ confidence interval (CI) throughout) in the population-weighted average of ambient (outdoor) $\mathrm{PM}_{2.5}$ concentrations between P1 (64 (57-70) $\left.\mathrm{gg} \mathrm{m}^{-3}\right)$ and P2 (47 (41-54) $\left.\mathrm{gg} \mathrm{m}^{-3}\right)$. In contrast, the population-weighted exposure (PWE) that considers both indoor and ambient concentrations shows an increase of $5.7 \mu \mathrm{g} \mathrm{m}^{-3}\left(1.2-11.0 \mu \mathrm{g} \mathrm{m}^{-3}\right)$ in $\mathrm{P} 2\left(101(87-117) \mu \mathrm{g} \mathrm{m}^{-3}\right)$ compared to P1 (95(83-109) $\mathrm{kg} \mathrm{m}^{-3}$ ) (Fig. 1), suggesting important roles of other factors, including population migration and the time spent indoors, in the PWE change under the quarantine. Decomposition analysis, by changing the factors severally, attributes the changes of $-10.5(-12.8$ to -8.5$), 10.8(8.2-14.2)$ and $5.4(3.2-8.1) \mu \mathrm{g} \mathrm{m}^{-3}$ in PWE to the changes in ambient $\mathrm{PM}_{2.5}$, population migration and time spent indoors, respectively (Fig. 2). Note that changes in ambient $\mathrm{PM}_{2.5}$ affect indoor concentration through infiltration, which is included in our assessment. Population migration alone offsets the effect of the ambient air quality improvement on PWE $\left(10.8 \mu \mathrm{g} \mathrm{m}^{-3}\right.$ due to migration versus $-10.5 \mu \mathrm{g} \mathrm{m}^{-3}$ due to ambient air) (Fig. 2).

The effects of migration on PWE. The dynamic cross-province migration dataset we established is based on the national census data ${ }^{26}$ and official reports ${ }^{14}$ and is temporally allocated using the Baidu real-time mobility data ${ }^{27}$ (Methods). The direction of the migration is characterized on a province-to-province basis and further divided into four categories: (1) urban-to-rural, (2) urban-to-urban, (3) rural-to-rural, and (4) rural-to-urban. The migration started about 25 days before the Spring Festival and had two phases with opposite directions-one occurred in P1, the other in P2. Before the Spring Festival (P1), there were estimated 236 million people returning to their hometowns, accounting for one-sixth of the total population. Urban-to-rural migration contributed $53 \%$ of the total, of which most were reportedly rural migrant workers ${ }^{14}$. Urban-to-urban migration contributed $34 \%$ and other two types of migration were relatively minor (10\% for rural-to-rural and $3 \%$ for rural-to-urban). After the Spring Festival (P2) when people would normally move back to cities, however, the nation was under quarantine in response to the outbreak of COVID-19 and the migration froze. The effect of the quarantine on the migration in P2 is clearly illustrated by the day-by-day comparison in the migration intensity in 2020 with the previous year (Fig. 3). The migration in P2 was close to completion within 25 days after the 2019 Spring Festival, by which time this year the migration was only $18 \%$ complete (Fig. 3 ).

The migration led to a shift in the fraction of population residing in rural areas. The fraction reached its maximum of $47.6 \%$ during the Spring Festival as in normal years but decreased at a pace one-seventh that of normal years afterwards due to the quarantine ( $0.05 \%$ per day in 2020 versus $0.34 \%$ per day in 2019 ; Fig. 3 ).

Two main consequences of such a change in the migration for population exposure were: (1) a larger fraction of people exposed for a longer time to HAP in rural households which is usually more severe than in urban households ${ }^{13,18}$; and (2) increased rural energy consumption to meet the demand of the immigrants, both of which further worsened HAP. On the basis of a recently conducted national survey on rural household energy consumption ${ }^{19}$ and the indoor exposure model ${ }^{10,12}$ (Methods), we estimate that by increasing the fraction of rural population, the migration enhanced the nationwide PWE by $3.1(2.3-4.0) \mu \mathrm{g} \mathrm{m}^{-3}$ and $7.7(5.9-10.0) \mu \mathrm{g} \mathrm{m}^{-3}$ in P1 and P2, respectively, compared to a baseline scenario assuming no migration (Fig. 2), while by increasing the household energy consumption, the migration further increased the PWE by 3.6 (2.74.6) $\mu \mathrm{g} \mathrm{m}^{-3}$ and $9.7(7.4-12.5) \mu \mathrm{g} \mathrm{m}^{-3}$, respectively, in $\mathrm{P} 1$ and $\mathrm{P} 2$ (Fig. 2). This amounts to total increases of $6.6(5.0-8.6) \mu \mathrm{g} \mathrm{m}^{-3}$ and

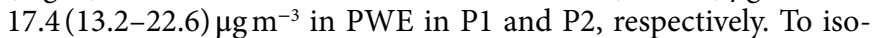
late the impact of the COVID-19-induced freezing of the migration on PWE, we substitute migration in 2019 for that experienced in 2020 while keeping all other factors equal (outdoor air quality, time spent indoors, baseline energy mix, and so on). The results show 


\begin{tabular}{|l|}
\hline PWE change due to changes in \\
A - Ambient air quality \\
R - Population relocation \\
E - Household energy consumption \\
T - Time spent indoors \\
\hline
\end{tabular}

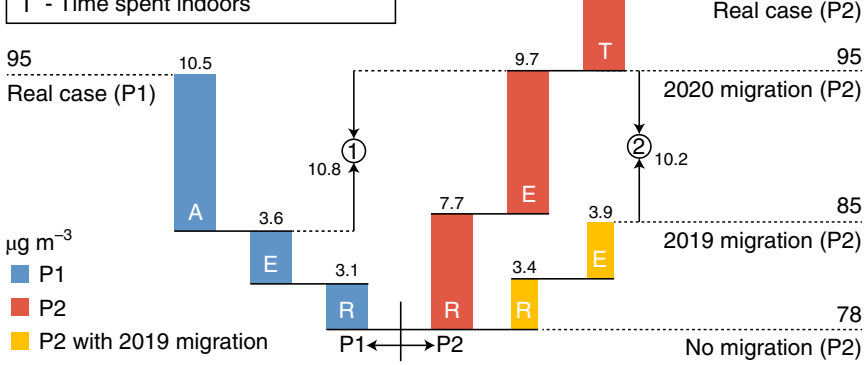

Fig. 2 | Decomposition analysis of the PWE change between P1 and P2. The overall change in the PWE of the Chinese population is decomposed into the changes in PWE due to the changes in ambient air quality, population relocation, household energy consumption and time spent indoors. Note that the migration had two phases with opposite directions: the first one (during P1) preceded the Spring Festival when people returned to their hometowns; the second (during P2) followed the first as people travelled back to work. The quarantine froze the second phase of the migration, leading to a net difference in the migration impact on PWEs between P1 and P2, as marked in the figure. The impact of the quarantine-induced freezing of the migration in response to COVID-19 in P2 is evaluated by comparing with the PWE under 2019 migration pattern and is also marked in the figure. PWEs are in $\mu \mathrm{g} \mathrm{m}^{-3}$.

a comparable increase in PWE in $\mathrm{P} 1\left(6.5 \mu \mathrm{g} \mathrm{m}^{-3}\right.$ in 2019 versus $6.6 \mu \mathrm{g} \mathrm{m}^{-3}$ in 2020) but a much smaller increase in P2 (7.2 versus $\left.17.4 \mu \mathrm{g} \mathrm{m}^{-3}\right)$, suggesting an enhancement of $10.2(7.7-13.3) \mu \mathrm{g} \mathrm{m}^{-3}$ (17.4 minus $7.2 \mu \mathrm{g} \mathrm{m}^{-3}$ ) in PWE due to the freezing of the migration under the national quarantine.

The contribution of HAP on PWE and the inequality of the PWE change. Focusing on the quarantine period (P2), we consider the changes in HAP and other sectors (transportation, industry and power generation) and assess the overall impacts of the quarantine on indoor and ambient air quality and on PWE. Our assessment shows an estimated decrease of $15.6(10.0-20.6) \mu \mathrm{g} \mathrm{m}^{-3}$ in the population-weighted average ambient $\mathrm{PM}_{2.5}$ due to the quarantine, which is similar in magnitude to the $\mathrm{PM}_{2.5}$ reduction before and after the COVID-19 outbreak $\left(16.7 \mu \mathrm{g} \mathrm{m}^{-3}\right)$ (Fig. 4). We note, however, that unlike our fused $\mathrm{PM}_{2.5}$ field which is the best guess of the real-world $\mathrm{PM}_{2.5}$ concentrations, our impact assessment on ambient $\mathrm{PM}_{2.5}$ using chemical transport model is limited by the uncertainty in the estimation of quarantine-induced emission reduction (Methods) and the capability of the model to reproduce the actual $\mathrm{PM}_{2.5}$ change in response to the emission reduction ${ }^{28}$, both of which warrant further investigation. The indoor $\mathrm{PM}_{2.5}$ concentration is estimated to increase by $3.1(-2.4-8.8) \mu \mathrm{g} \mathrm{m}^{-3}$ due to the quarantine (Fig. 4b) which is a result of the competition between the exacerbation of HAP $\left(12.2 \mu \mathrm{g} \mathrm{m}^{-3}\right)$ and the improvement in ambient $\mathrm{PM}_{25}$ that infiltrates indoors $\left(-9.1 \mu \mathrm{g} \mathrm{m}^{-3}\right)$. Incorporating the changes in indoor and ambient $\mathrm{PM}_{2.5}$ with population migration and human activities, we estimate that the COVID-19 quarantine led to a net

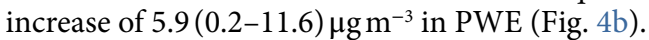

We calculate the contribution of HAP on PWE, which includes the direct contributions to indoor and outdoor $\mathrm{PM}_{2.5}$ and the indirect contribution of outdoor HAP to indoor $\mathrm{PM}_{2.5}$ through infiltration (Supplementary Fig. 1). HAP dominated the PWE in P2 regardless of whether there was a quarantine, whereas the COVID19-induced quarantine increased the HAP contribution to PWE from $74 \%$ (no quarantine or no COVID-19) to $82 \%$ (in the real case) (Supplementary Fig. 2).

The contribution of HAP to PWE during this period was higher than that before the COVID-19 quarantine (68\%), or in a counterfactual scenario where there was no migration (70\%), or for the annual average (62\%) (Supplementary Fig. 2). The leading cause of HAP is the use of solid fuels (for example, coals and biomass) for cooking and heating, which is much more prevalent in rural areas $(67.5 \%$ as the share of solid fuels in the household energy mix) than in urban areas (4.7\%) (Fig. 4c). Further investigation shows a clear tendency toward a stronger positive effect of the quarantine on PWE as the share of solid fuel use increased (Fig. 4b,c and Supplementary Fig. 3) and that the PWE in rural areas was estimated to increase by 19.2 (13.5-25.6) $\mu \mathrm{g} \mathrm{m}^{-3}$ due to the quarantine, while the urban PWE decreased by $14.0(8.6-20.7) \mu^{-3} \mathrm{~m}^{-3}$.

The change in exposure associated with solid fuel use and the contrary changes in rural and urban PWEs are due primarily to the interaction between HAP and the human activities: the longer time spent indoors during the pandemic increased the time length for people being exposed to HAP and thus increased the PWE among rural residents; the freezing of the migration increased the rural household energy consumption and subsequently increased the severity of HAP. On the other hand, in urban areas where indoor air quality is often better than outdoor ${ }^{29}$, the increase in the time spent indoors reduced PWE.

The association between PWE and HAP led to the spatial heterogeneity (Fig. 5) and population inequality in the quarantine-induced changes in PWE (Fig. 4c) which ranged from $-19.0 \mu \mathrm{g} \mathrm{m}^{-3}$ in Tianjin to $32.5 \mu \mathrm{g} \mathrm{m}^{-3}$ in Inner Mongolia and from $-9.5 \mu \mathrm{g} \mathrm{m}^{-3}$ in the provinces with average per-capita incomes higher than US $\$ 5,000$ to $6.5 \mu \mathrm{g} \mathrm{m}^{-3}$ in the provinces with average per-capita incomes lower than US $\$ 3,000$, suggesting unequal changes in PWE by income group. The inequality in the PWE changes is further confirmed by the significant negative correlation between the PWE changes and provincial per-capita income levels $(P<0.001)$ and survives the assessments using county-level data or focusing on the rural population exclusively (Supplementary Fig. 4a,b).

The urban population does not show significant inequality (Supplementary Fig. 4c) probably due to the much lower dependence on solid fuels and therefore being less affected by HAP than their rural counterparts. Regression analysis reveals a significant interaction (the regression coefficient of the interaction term is -0.69 $(-0.84$ to -0.54$), P<0.001)$ between the per-capita income and the epidemic severity in the model to predict the quarantine-induced changes in PWE (Methods and Supplementary Table 2) and suggests that regions with more severe epidemic situation are associated

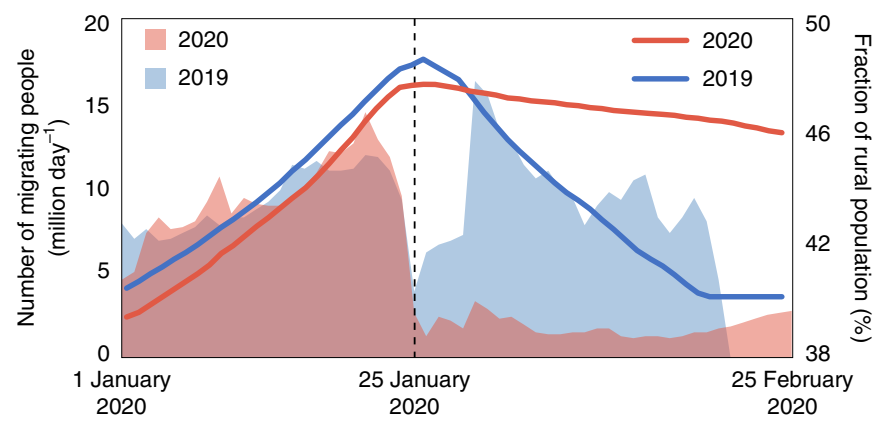

Fig. 3 | The population migration around the Spring Festivals of 2019 and 2020. The shaded areas illustrate the temporal trend of the number of people migrating each day. The solid lines show the temporal trends of the fraction of rural population (the population residing in rural areas) in the total population. The black dashed line marks the Spring Festival. The $x$ axis represents the calendar date in 2020. 

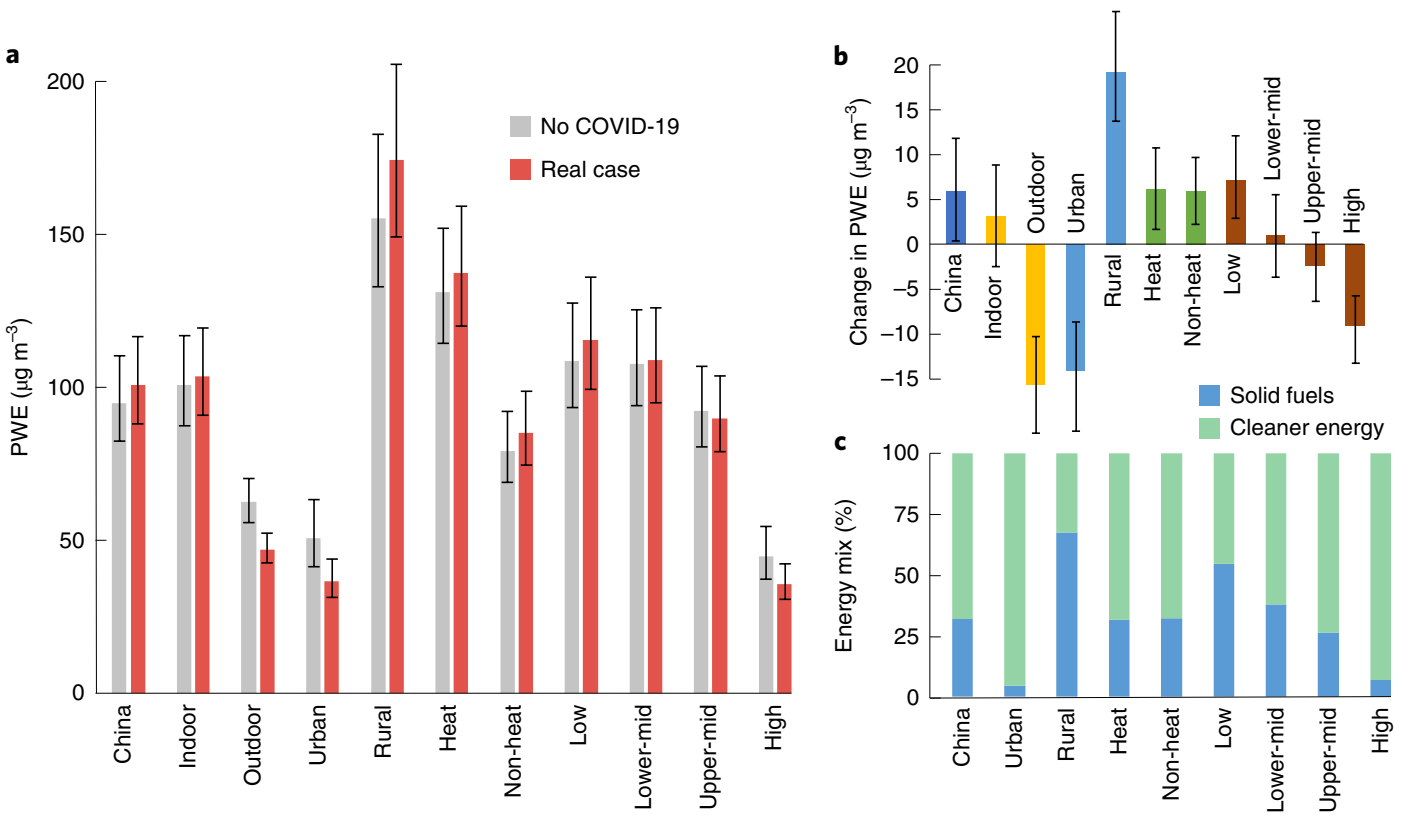

Fig. 4 | The impacts of the responses to COVID-19 on PWEs and the use of solid fuels as a driving factor. a, The PWEs in the real case and in the 'no COVID-19' scenario. b, The changes in PWEs due to the responses to COVID-19. c, The shares of solid fuel use in household energy mix. Panels a-c show effects in China, in indoor vs outdoor environments, in urban vs rural areas, in heating vs non-heating regions and in provinces with different per-capita income levels. The shares of solid fuel use in household energy mix in indoor vs outdoor environments are the same as in national total and thus are not shown in $\mathbf{c}$. Error bars in $\mathbf{a}$ and $\mathbf{b}$ represent $95 \% \mathrm{Cls}$.

with greater inequality. In Hubei, every $20 \%$ reduction in income is estimated to be associated with an increase of $6.7(5.9-7.5) \mu \mathrm{g} \mathrm{m}^{-3}$ in PWE $(P<0.001)$ due to the quarantine, which is almost twice the increase for the national average, $3.4(3.2-3.5) \mu \mathrm{g} \mathrm{m}^{-3}(P<0.001)$ (Supplementary Fig. 4a and Supplementary Table 2).

The effect of Clean Heating Plan on PWE changes. Despite the heterogeneity and inequality, the quarantine-induced increases in PWE in the heating (north) and non-heating (south) regions, including both rural and urban areas, were comparable-6.2(1.6-

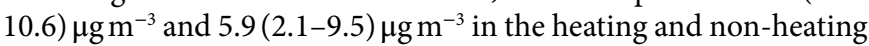
regions, respectively (Figs. $4 \mathrm{~b}$ and 5 ). We find that a recently implemented campaign called Clean Winter Heating Plan in Northern China (Clean Heating Plan for short), played an important role in balancing the PWE increases between heating and non-heating regions. Clean Heating Plan was launched by the Chinese central government in 2017 and set stringent and differentiated goals through 2021 toward a high rate of clean heating (the rate of clean energy used for heating) in the northern region, with the rates ranging from $40 \%$ in rural areas to $100 \%$ in some major cities ${ }^{30}$. This campaign, if successfully implemented, would reduce the amount of annual coal consumption by $150 \mathrm{Tg}$, and recent progress has shown much success in the implementation of this campaign such that it is expected to be achieved ahead of schedule ${ }^{31}$.

We estimate that Clean Heating Plan had phased out $44.4 \%$ of the solid fuels used in households in the northern provinces by the end of 2019. If there was no such campaign, we estimate that the increase in PWE induced by COVID-19 would be almost doubled in the heating region $\left(12.0 \mu \mathrm{g} \mathrm{m}^{-3}\right)$. In addition, the population inequality in the PWE increase, measured by the increase in PWE per $20 \%$ reduction in income $\left(4.6(4.3-4.9) \mu \mathrm{g} \mathrm{m}^{-3}\right)$, would be $30.1 \%$ higher than is estimated in the real case $\left(3.5(3.2-3.8) \mu \mathrm{g} \mathrm{m}^{-3}\right)$ in the heating region (Supplementary Fig. 5 and Supplementary Table 2). In an ideal case where Clean Heating Plan was fully phased in, the quarantine would only lead to an increase of $2.3 \mu \mathrm{g} \mathrm{m}^{-3}$ in
PWE in the heating region, with the inequality decreased by $15.6 \%$ (Supplementary Fig. 5 and Supplementary Table 2). Our analysis thus reveals that Clean Heating Plan moderated the quarantine-induced increases in PWE in the heating region, reduced the inequality of the PWE increases among different income groups of people, and put the PWE increases of the heating and non-heating regions in the balance. Still, the PWE in the heating region $\left(137 \mu \mathrm{g} \mathrm{m}^{-3}\right)$ was $61 \%$ higher than it was in the non-heating region $\left(85 \mathrm{\mu g} \mathrm{m}^{-3}\right)$ and, in rural areas, the quarantine-induced increase in PWE in the heating region $\left(24.4 \mu \mathrm{g} \mathrm{m}^{-3}\right)$ was $31 \%$ higher than in the non-heating region $\left(18.6 \mu \mathrm{g} \mathrm{m}^{-3}\right)$.

\section{Discussion}

In this study, we integrate multiple data sources and modelling techniques to dynamically track the changes in PWE due to the national quarantine in China. We first show that the national population-weighted exposure to ambient $\mathrm{PM}_{2.5}$ reduced by $16.7 \mu \mathrm{g} \mathrm{m}^{-3}$ but the average PWE of the population considering both indoor and outdoor $\mathrm{PM}_{2.5}$ exposure is estimated to increase, which is mainly due to the worsened HAP and a higher opportunity for people to be exposed to indoor HAP during the pandemic. Changes to the actual dose of $\mathrm{PM}_{2.5}$ to the population will also depend on changes in use and effectiveness of facemasks during the period ${ }^{32,33}$, which deserves further studies.

With respect to the distribution of PWE, our assessment reveals an increase in the environmental inequality of air pollution exposure in response to the COVID-19 crisis. While the high-income group benefited from the reduction of PWE, the low-income group suffered a significant increase in PWE. Such inequality would be even higher if Clean Heating Plan that targets HAP in northern China was not implemented. In addition, given the reported association between short-term exposure to air pollution and the transmission of COVID-19 (ref. ${ }^{23}$ ), this analysis shows how the COVID19 pandemic itself, as well as the quarantine, may have deepened health inequalities. Our assessment highlights the importance of 


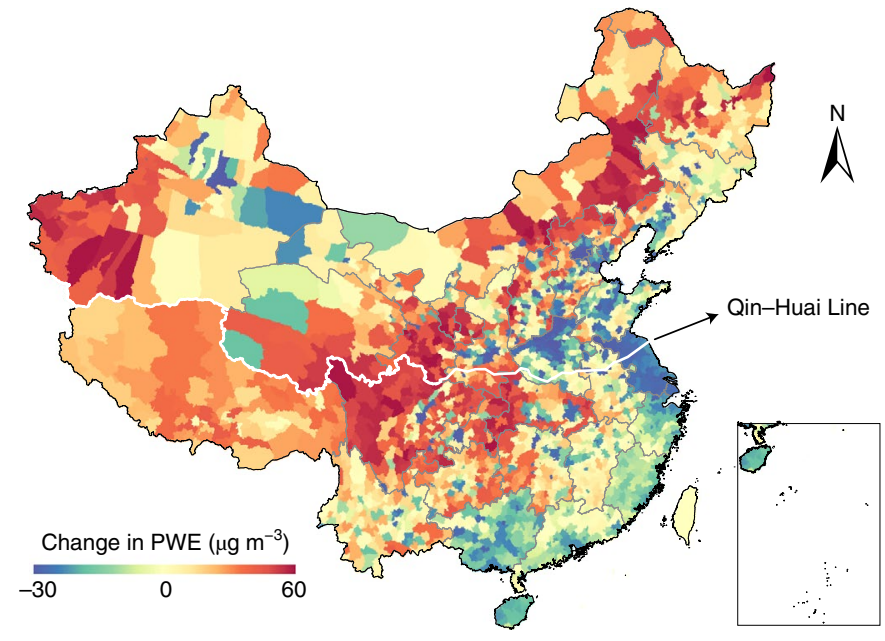

Fig. 5 | The spatial distribution of the changes in PWE among the Chinese population due to responses to COVID-19. Changes in ambient and indoor air quality, population migration and time spent indoors are considered. The PWE changes are illustrated by county. The white line marks China's Qinling Mountains-Huai River Line (Qin-Huai Line) Qin-Huai Line divides China into two regions that differ in climate and is commonly used as a reference line in policy-making to determine the heating (northern) and non-heating (southern) regions ${ }^{56}$. The map of China map is from Harvard Dataverse and is publicly available under the Creative Commons CCO Public Domain Dedication ${ }^{57}$.

mitigating HAP for reducing the environmental inequality and protecting human health. If society is to confine people to their homes for their protection, it is far better that they have clean heating to start with.

Our study has several limitations. The emission changes in response to the COVID-19 quarantine are modelled implicitly by assuming constant reduction rates for individual sectors across the nation. Although the resulting changes in CMAQ-modelled outdoor $\mathrm{PM}_{2.5}$ concentrations show general agreement with observation-based $\mathrm{PM}_{2.5}$ changes (data fusion), the model simulation based on such implicit emission estimates is probably subject to large uncertainty. To better reproduce the spatial heterogeneity in the air quality response, subnational-level information on energy consumption, traffic intensities and industrial activities needs to be incorporated in the emission estimation. Note that compared to the CMAQ simulation, the $\mathrm{PM}_{25}$ fields derived from the data fusion approach show relatively small uncertainty (Supplementary Methods 1 and 2 and Supplementary Figs. 6 and 7).

Notably, our uncertainty analysis identifies the HAP-induced indoor $\mathrm{PM}_{2.5}$ assessment as the predominant source of uncertainty in PWE (Supplementary Methods 3 and Supplementary Figs. 8 and 9). This large uncertainty associated with HAP is introduced by uncertainties in rural household energy consumption and indoor $\mathrm{PM}_{2.5}$ concentrations, due primarily to the limited availability of such data in China. We show that HAP plays a central role in PWE during both normal periods (contributing $>60 \%$ of the total PWE) and the quarantine period (80\%). Nationwide surveys on household energy consumption and large-scale indoor air quality monitoring networks in rural China (Supplementary Figs. 8 and 10) may provide necessary information to narrow down the uncertainty in PWE. The importance of HAP in overall exposure, the differentiated responses to the quarantine between different population groups, and the large uncertainty in indoor $\mathrm{PM}_{2.5}$ assessment indicate that a better understanding of HAP is probably instrumental in improving air quality while maintaining environmental equity of the Chinese population.

\section{Methods}

Household energy consumption. Provincial-level household energy consumption data for 1992 to 2012 were collected and compiled on the basis of a representative national survey ${ }^{19}$ and China Statistical Yearbook ${ }^{34}$. The data were downscaled to county level and extrapolated to 2020 (the study year) on the basis of the fuel type-specific empirical models developed by Shen et al. ${ }^{35,36}$. Following a previous study ${ }^{10}$, the clean heating targets set by Clean Heating Plan were incorporated into the energy trends in the heating region.

Migration data. We derived detailed origin and destination information from the sixth National Census ${ }^{26}$ to characterize population migration on the county level ${ }^{36}$. The census data classified the migrants into four groups (rural-to-urban, urban-to-urban, rural-to-rural and urban-to-rural) and are representative of the migration pattern in 2010. The census data showed a total of 138 million migrant workers in 2010, noting that not all the migrants intended to return home during the Spring Festival holidays. The Ministry of Human Resources and Social Security reported 125 million migrant workers returning home in $2020^{14}$. Therefore, the census data was scaled down by a factor of 0.9 to represent the migration pattern in 2020. The Spring Festival migration can be divided into two phases with opposite directions. In the first (back-home) phase, migrants go back home to reunite with their families and celebrate the upcoming New Year; in the second (returning-to-work) phase, migrants return to work. The first phase typically ends by New Year's Eve (that is, the end of the old year and the start of the Spring Festival holidays), followed by the second phase. However, there is a small fraction of people who get back home after New Year's Eve, which yields a temporal overlap between the first and second phases. We therefore assumed that the back-home migration was achieved before the second day of the Spring Festival holidays, and that the returning-to-work migration started from the first day of the Spring Festival holidays. The migration flows (the number of migrants) were temporally allocated using the daily cross-province mobility intensities reported by the Baidu real-time mobility monitoring platform as a surrogate ${ }^{27}$. Our assessment showed that the migrants who got back home after New Year's Eve only occupied $1.6 \%$ of the total migrants in the first phase. For the 2019 Spring Festival, of which the detailed provincial-level Baidu mobility data were not available, the national-level mobility intensities were used to scale the 2020 migration pattern to 2019 , assuming that the relative difference in the migration flows across provinces remained unchanged between 2019 and 2020.

Survey on human activity pattern. The information on the daily time spent indoors and in different indoor compartments (kitchen, living room and bedroom) in wintertime were derived from Exposure Factors Handbook of Chinese Population $^{37}$, as summarized by Chen et al. ${ }^{12}$, and used in this study to represent the time-activity pattern when there was no COVID-19. The time-activity patterns during the pandemic were derived from an online questionnaire survey (https:// www.wjx.cn/m/59666734.aspx) which collected information on the frequencies of going out during the quarantine together with demographic information, including gender, age, urban/rural residents, highest level of education, province/city and occupation $^{38}$. To answer the question on frequencies of going out during the quarantine period, participants were asked to choose one of the following options: (1) more than twice a day, (2) once a day, (3) once per 2 days, (4) once per 3 days, (5) once per 4 days, (6) once per 5 days, (7) once per 6 days, (8) once a week, (9) less frequent than once a week or (10) never.

This survey adopted strict quality control measures during data processing and analysis $^{38}$. The questionnaires with missing values, logical errors and data format errors were excluded. Two groups of personnel independently derived the data and completed the comparison to ensure the accuracy of the results. A total of 8,330 questionnaires were distributed with a recovery rate of $100 \%$. A total of 7,784 valid questionnaires were obtained, covering 31 provinces in China ${ }^{38}$.

Among the participants, 3,364 were males and 4,420 were females. There were 183 participants at an age of less than $18 \mathrm{yr}, 4,646$ between 18 and $30 \mathrm{yr}$, 1,920 between 45 and $60 \mathrm{yr}$ and 103 older than $60 \mathrm{yr}$. Information on going-out frequencies by gender and age was provided on Open Science Framework at https://osf.io/x46tb/. Overall going-out frequencies were adjusted by the gender and age distributions of the general Chinese population. The survey showed that the more severe the epidemic, the less frequently people went out each day. Going-out frequencies were translated into time lengths of outdoor stay by assuming the time length for each going-out event ranging from $200 \mathrm{~min}$ in the provinces that were the least affected by the COVID-19 outbreak (Qinghai and Tibet) to $120 \mathrm{~min}$ in Hubei where the outbreak was the most severe. The uncertainty induced by this assumption was considered in the uncertainty analysis specified in following section. The average time spent indoors by province before and during the pandemic is summarized in Supplementary Table 1.

Emissions and air quality modelling. We used AiMa emission inventory ${ }^{39,40}$ as the emission input to conduct the air quality modelling for ambient $\mathrm{PM}_{2.5}$ assessment. The emission inventory has been compiled by integrating a variety of sources (for example, statistical data and emission inventories $)^{40}$ and has undergone continuous updates. This inventory is currently used by the online operational AiMa system (http://www. aimayubao.com/) that provides air quality forecast for the Chinese government and public. The base year of the latest version of the AiMa inventory is 2017. 
The ambient $\mathrm{PM}_{2.5}$ concentrations were obtained by combining hourly ground-level observations reported by the China National Urban Air Quality Real-time Publishing Platform ${ }^{5}$ with model predictions by the Community Multiscale Air Quality (CMAQ) model v.5.0.2 (ref. ${ }^{41}$ ) using an ensemble deep learning data fusion approach ${ }^{25}$. Meteorological variables were derived from the AiMa system, which were generated by the Weather Research Forecasting (WRF) model v.3.4.1 (ref. ${ }^{42}$ ) driven by the 0.5 -degree global weather forecast products produced by the National Centres for Environmental Prediction Global Forecast System $^{43}$. The downscaled meteorology together with the AiMa emission inventory was used to drive CMAQ simulation which was conducted to cover the mainland China on a horizontal resolution of $12 \mathrm{~km}$ with 13 vertical layers extending up to $\sim 16 \mathrm{~km}$ above ground. It should be noted that our CMAQ simulation was conducted on a $12-\mathrm{km}$ horizontal resolution, primarily due to the resolution in the emissions inventory, and that $\mathrm{PM}_{2.5}$ and ozone fields are typically relatively homogeneous spatially ${ }^{44,45}$. Further, the computational costs of large-scale simulations on a finer resolution would be high. The model output was fused with observations from the China National Environmental Monitoring Centre (http://www.cnemc.cn) to get the final ambient $\mathrm{PM}_{25}$ concentration fields across China on a daily resolution over the study period (from 25 December 2019 to 25 March 2020). We compared the fused data with observations from an independent dataset and found a good agreement between the two sets of data in terms of the spatial distribution and temporal trends (Supplementary Figs. 6 and 7). This evaluation confirmed the good performance of the data fusion approach in reproducing ground-level $\mathrm{PM}_{25}$ concentrations. Details are provided in Supplementary Methods 1 and Supplementary Figs. 6 and 7. More information on the emission inventory, the model configuration, and the data fusion procedures can be found in a previous study ${ }^{25}$.

We conducted adjoint analysis to decompose the contributions of various emission sources to outdoor $\mathrm{PM}_{25}$ concentrations. The emission sources, as categorized in the AiMa inventory, included power generation, industry, residential (household), transportation, agriculture, solvent usage, fugitive dust and fires. CMAQ-Adjoint v.5.0 (ref. ${ }^{46}$ ) was applied to calculate the adjoint sensitivities. The adjoint analysis provides location- and time-specific gradients (adjoint sensitivities) and can be used in applications such as backward sensitivity analysis, source attribution, optimal pollution control, data assimilation and inverse modelling ${ }^{46}$. The CMAQ-Adjoint v.5.0 is the most up-to-date version of CMAQ-Adjoint. Discrete adjoint is implemented for gas-phase chemistry, aeroso formation, cloud chemistry and dynamics, and diffusion. Continuous adjoint is implemented for advection. The model performance has been comprehensively evaluated in the previous study ${ }^{46}$, showing good agreements with the results given by forward sensitivity analysis.

In this study, the cost function of the adjoint analysis was defined as the ambient population-weighted average $\mathrm{PM}_{2.5}$ concentration over the study period across China. The adjoint model thus provided sensitivities of this cost function to per-unit emissions of various species in each model grid cell. Using the source-specific emission information, we evaluated the source contributions of household (residential) energy consumption and other sectors on ambient air pollution by province. Details about the principle equations, development and evaluation of CMAQ-Adjoint can be found in previous studies ${ }^{46,47}$.

Using the adjoint sensitivities, we further evaluated the changes in the population-weighted concentration in response to the emission reduction during the quarantine. Huang et al..$^{28}$ estimated sector-specific emission reductions due to the COVID-19 quarantine in China on the basis of dynamic economic and industrial activity levels. The description on the emission reduction estimation was detailed in Huang's study ${ }^{28}$. Following Huang's study, we adopted a reduction rate of $10 \%$ in power plant emissions, $30 \%$ in industrial emissions and $70 \%$ in mobile emissions. The changes in residential emissions due to population migration were evaluated using the procedures as specified in our previous studies ${ }^{35,36}$.

Indoor exposure model. We used an indoor exposure model developed by Chen et al. ${ }^{12}$ to quantify the indoor $\mathrm{PM}_{25}$ levels. The model was modified to take into account the change in the amount of household energy consumption and outdoor infiltration and to unify the estimation approach for urban and rural household conditions as follows

$$
C_{\text {in }}=C_{\text {in }, \text { add }}+C_{\text {out,add }}
$$

where $C_{\text {in }}$ is the indoor $\mathrm{PM}_{2.5}$ concentration in $\mu \mathrm{g} \mathrm{m}^{-3}, C_{\text {in,add }}$ is the $C_{\text {in }}$ component contributed by indoor sources and $C_{\text {out add }}$ is the $C_{\text {in }}$ component contributed by outdoor infiltration. The value of $C_{\text {in,add }}$ was calculated by the following equation:

$$
C_{\text {in }, \text { add }}=\frac{\sum E_{f} \times C_{f, k} \times T_{k}}{\bar{E} \times \sum T_{k}}
$$

where subscripts $f$ and $k$ denote the type of fuel (wood, straw, coal and cleaner energy) and indoor compartment (kitchen, living room and bedroom), respectively; $E_{f}$ is the per-household daily consumption of fuel type $f$ in terms of thermal energy amount (the amount of energy consumption after thermal efficiency conversion); $\bar{E}$ is the average per-household daily thermal energy required for cooking and heating; $C_{f, k}$ is the $C_{\text {in,add }}$ in indoor compartment $k$ when $E_{f}=\bar{E}$ and the household consumes fuel $f$ solely; $T_{k}$ is the time spent daily in indoor compartment $k$. Following a previous study ${ }^{48}$, the thermal efficiencies of biomass, coal, gas and electricity are $0.154,0.244,0.555$ and 0.84 , respectively. The $\bar{E}$ value $40 \mathrm{MJ} \mathrm{day}^{-1}$ household ${ }^{-1}$ was calculated as the national average daily household thermal energy consumption for cooking and heating in winter. Values for $C_{f k}$ were adopted from a previous study ${ }^{12}$ in which the means and variations of $\mathrm{C}_{f, k}$ were determined by a meta-analysis of 28 field measurement studies in China (see https://osf.io/x46tb/ for the complete dataset). The mean heating-season $C_{f, k}$ in kitchen/living room are 283, 434 and $547 \mu \mathrm{g} \mathrm{m}^{-3}$ for coal, crop and wood, respectively, and in bedroom are $211,267,359 \mu \mathrm{g} \mathrm{m}^{-3}$ for coal, crop and wood, respectively. Cleaner energy was assumed to cause little addition to indoor $\mathrm{PM}_{25}$ and thus the $C_{f k}$ for cleaner energy was set to be 0 . Equation (2) assumes that with all others being equal, $C_{\text {in,add }}$ is proportional to the thermal amount of daily energy consumption of the household. This assumption was testified and supported by sensitivity tests using a single-box $\operatorname{model}^{49}$, as recommended in World Health Organization's indoor air quality guidelines ${ }^{50}$, to predict $C_{\text {in,add }}$ on the basis of varying amounts of energy consumption. The value $C_{\text {out,add }}$ was calculated by multiplying ambient $\mathrm{PM}_{2.5}$ concentrations with region-specific infiltration factors following the method of Xiang et al..$^{51}$. The modelled temporal trend in indoor $\mathrm{PM}_{2.5}$ concentrations captured observed temporal trends measured in 30 households in a rural area of Shandong province during the study period (Supplementary Fig. 10). The $\mathrm{PM}_{2.5}$ exposure of individuals at a specific location was calculated as the average of the indoor and outdoor $\mathrm{PM}_{2.5}$ concentrations weighted by the time fractions of indoor and outdoor stays. The calculated exposure was evaluated against personal exposure measurements reported in the literature (Supplementary Fig. 8), which showed general agreement. The PWE in a region was calculated as the population-weighted average of the individuals' exposure within this region. The same approach to calculating PWE has been adopted in previous studies ${ }^{10,11}$.

Regression analysis. We conducted regression analysis to predict the county-level quarantine-induced changes in PWE. The regression showed significant interaction between per-capita income and the epidemic severity. The regression equation including the interaction term is as follows

$$
\mathrm{dPWE}=-31.9 \times \ln \left(\mathrm{INC}_{\mathrm{per}}\right)-0.69 \times \mathrm{SEV} \times \ln \left(\mathrm{INC}_{\mathrm{per}}\right)+124.6
$$

where dPWE denotes the change in PWE due to the COVID-19-induced quarantine in $\mu \mathrm{g} \mathrm{m}^{-3}$; $\mathrm{INC}_{\text {per }}$ is per-capita annual income in US\$; SEV is the epidemic severity determined by the confirmed cases in the provinces (Supplementary Table 1), ranging from 1 in Qinghai and Tibet (the least severe) to 5 in Hubei (the most severe). In addition to equation (3), we developed single models (excluding the interaction term) to investigate the relationship between per-capita income and dPWE for specific regions (for example, Hubei, Northern China and urban/rural areas) and for different counterfactual cases (for example, assuming no Clean Heating Plan or assuming that Clean Heating Plan has been fully phased in). See Supplementary Table 2 for the full model results, including coefficients, standard errors and $P$ values of individual models.

Uncertainty analysis. The uncertainty in the PWE estimates stemmed from various sources, including the uncertainties in the modelled ambient and indoor concentrations, population migration, time-activity patterns and infiltration factors. We conducted Monte Carlo simulations to propagate the uncertainties from these input sources to PWE. For some input variables (for example, concentration, migration intensity and time spent indoors), we assumed log-normal distributions to avoid negative values and used geometric coefficient of variation $(\mathrm{GCV})^{52}$ instead of coefficient of variation $(\mathrm{CV})$ to measure the uncertainty. GCV is defined as follows:

$$
\mathrm{GCV}=\sigma_{\mathrm{g}}-1
$$

where $\sigma_{\mathrm{g}}$ is the geometric standard deviation ${ }^{53}$. Variable uncertainties measured by the same value of $\mathrm{CV}$ and GCV have similar widths of uncertainty intervals. For example, the uncertainties with CV and GCV of $20 \%$ have semi-interquartile ranges (half of the difference between the upper quartile and the lower quartile) equal to $13.6 \%$ and $13.5 \%$ of the variable's mean, respectively, and have $95 \% \mathrm{CI}$ widths equal to $79 \%$ and $80 \%$ of the variable's mean, respectively.

The GCVs of fused ambient $\mathrm{PM}_{2.5}$ concentrations were derived on the basis of the performance of the data fusion approach (Supplementary Methods 2). The GCV differed by region and urban/rural area, depending on the region-specific performance of the data fusion approach and the number of monitoring sites available within each region (Supplementary Methods 2). The GCV of the population migration intensity was assumed to be $20 \%$. The GCV of the time spent indoors in normal days was set to be $5 \%$, following Chen et al. ${ }^{12}$. We assigned a CV (instead of GCV) of $20 \%$ to the changes in the time spent indoors due to the quarantine, thus resulting in a larger uncertainty in the time spent indoors during the quarantine period than in normal days.

For $\bar{E}$, we assumed a uniform distribution with a variation interval of $20 \%$ which is usually applied to reflect the uncertainty in the statistics of household solid fuel use $\mathrm{s}^{35,54}$. The $\mathrm{CV}$ of infiltration factors in indoor/outdoor air exchange was set to be $12.5 \%$ following Shi et al. ${ }^{55}$. The uncertainties in indoor $\mathrm{PM}_{2.5}$ 
concentrations in households using solid fuels were derived by Chen et al. on the basis of over 2,000 observations collected from the literature ${ }^{12}$. Given the large uncertainty in the estimated emission reduction due to the responses to COVID19 , CVs of the magnitudes of the emission reduction were set to be $30 \%$ for all sectors. GCVs were converted to standard deviations on the logarithmic scale by the follow equation

$$
\sigma_{\ln }=\ln (\mathrm{GCV}+1)
$$

where $\sigma_{\ln }$ is the standard deviation of a given parameter on the natural logarithmic scale and was used to determine probability distributions of corresponding parameters in Monte Carlo simulations for uncertainty analysis. Monte Carlo simulations were performed 1,000,000 times to propagate the uncertainties in input variables into the uncertainty in PWE

In addition, we investigated the contributions of various sources to the final uncertainty in PWE. The uncertainty sources we considered included the data fusion of ambient $\mathrm{PM}_{2.5}$, the HAP-related indoor $\mathrm{PM}_{2.5}$ estimation, the migration intensity, the time-activity pattern and the infiltration factors. The result showed that the indoor $\mathrm{PM}_{2.5}$ concentration is the most important source of uncertainty in the estimated national average PWE. Details can be found in Supplementary Methods 3 and Supplementary Fig. 9.

Reporting Summary. Further information on research design is available in the Nature Research Reporting Summary linked to this article.

\section{Data availability}

The population distribution data, the daily cross-province migration data, the daily ground-level $\mathrm{PM}_{2.5}$ fusion data, going-out frequencies by gender and age during the COVID-19 quarantine and all data used to generate the figures in the main text are openly available on Open Science Framework at https://osf.io/x46tb/. The provincial-level time-activity data used in this study are provided in Supplementary Table 1. Further information and requests should be directed to, and will be fulfilled by, the corresponding author.

\section{Code availability}

The CMAQ source code can be accessed at https://www.epa.gov/cmaq. Upon completion of expanded user testing, the CMAQ-Adjoint code will be hosted and distributed by US EPA.

Received: 9 June 2020; Accepted: 18 November 2020; Published online: 4 January 2021

\section{References}

1. Jiang, L., Wang, B., Qu, T. \& Liao, J. Thirty provinces activated first-level public health emergency response. Xinhua News http://www.xinhuanet.com/ politics/2020-01/25/c_1125502232.htm (25 January 2020).

2. Tian, H. et al. An investigation of transmission control measures during the first 50 days of the COVID-19 epidemic in China. Science 368, 638-642 (2020)

3. Wright, R. There's an unlikely beneficiary of coronavirus: the planet. CNN https://edition.cnn.com/2020/03/16/asia/china-pollution-coronavirus-hnk-intl/ index.html (17 March 2020).

4. Bauwens, M. et al. Impact of coronavirus outbreak on $\mathrm{NO}_{2}$ pollution assessed using TROPOMI and OMI observations. Geophys. Res. Lett. 47, e2020GL087978 (2020).

5. China National Urban Air Quality Real-Time Publishing Platform (China National Environmental Monitoring Center, 2020); http://www.cnemc.cn

6. He, G., Pan, Y. \& Tanaka, T. The short-term impacts of COVID-19 lockdown on urban air pollution in China. Nat. Sustain. https://doi.org/10.1038/ s41893-020-0581-y (2020).

7. Venter, Z. S., Aunan, K., Chowdhury, S. \& Lelieveld, J. COVID-19 lockdowns cause global air pollution declines. Proc. Natl Acad. Sci. USA 117, 18984-18990 (2020)

8. Zhao, Y. et al. Substantial changes in nitrogen dioxide and ozone after excluding meteorological impacts during the COVID-19 outbreak in mainland China. Environ. Sci. Technol. Lett. 7, 402-408 (2020).

9. Isaifan, R. The dramatic impact of Coronavirus outbreak on air quality: has it saved as much as it has killed so far? Glob. J. Environ. Sci. Manag. 6 275-288 (2020).

10. Meng, W. J. et al. Energy and air pollution benefits of household fuel policies in northern China. Proc. Natl Acad. Sci. USA 116, 16773-16780 (2019).

11. Zhao, B. et al. Change in household fuels dominates the decrease in PM2.5 exposure and premature mortality in China in 2005-2015. Proc. Natl Acad. Sci. USA 115, 12401-12406 (2018).

12. Chen, Y. L. et al. Estimating household air pollution exposures and health impacts from space heating in rural China. Environ. Int. 119, 117-124 (2018).

13. Smith, K. R. et al. Millions dead: how do we know and what does it mean? Methods used in the comparative risk assessment of household air pollution. Annu. Rev. Public Health 35, 185-206 (2014).
14. Press Conference held by the Ministry of Human Resources and Social Security to Introduce the Progress of the Human and Social Departments' Work related to the Epidemic (Central People's Government of the People's Republic of China, 19 March 2020); http://www.gov.cn/xinwen/2020-03/19/ content_5493239.htm

15. Big Data: The Travel Volume Predictions during Lunar New Year Holiday in 2020 (Ministry of Transport of the People's Republic of China, 14 January 2020); http://www.199it.com/archives/998165.html

16. Kraemer, M. U. et al. The effect of human mobility and control measures on the COVID-19 epidemic in China. Science 368, 493-497 (2020).

17. Traffic system works well in the seventh day of the Spring Festival holiday. Xinhua News http://www.xinhuanet.com/politics/2020-01/30/c_1125514185. htm (30 January 2020).

18. Zhang, J. J. \& Smith, K. R. Household air pollution from coal and biomass fuels in China: measurements, health impacts, and interventions. Environ. Health Perspect. 115, 848-855 (2007).

19. Tao, S. et al. Quantifying the rural residential energy transition in China from 1992 to 2012 through a representative national survey. Nat. Energy 3, 567-573 (2018).

20. Di, Q. et al. Association of short-term exposure to air pollution with mortality in older adults. JAMA 318, 2446-2456 (2017).

21. Dominici, F. et al. Fine particulate air pollution and hospital admission for cardiovascular and respiratory diseases. JAMA 295, 1127-1134 (2006).

22. Katsouyanni, K. et al. Short term effects of ambient sulphur dioxide and particulate matter on mortality in 12 European cities: results from time series data from the APHEA project. Brit. Med. J. 314, 1658 (1997).

23. Zhu, Y., Xie, J., Huang, F. \& Cao, L. Association between short-term exposure to air pollution and COVID-19 infection: evidence from China. Sci. Total Environ. 727, 138704 (2020).

24. Wu, X., Nethery, R. C., Sabath, B. M., Braun, D. \& Dominici, F. Air pollution and COVID-19 mortality in the United States: strengths and limitations of an ecological regression analysis. Sci. Adv. 6, eabd4049 (2020).

25. Lyu, B. et al. Fusion method combining ground-level observations with chemical transport model predictions using an ensemble deep learning framework: application in China to estimate spatiotemporally-resolved $\mathrm{PM}_{2.5}$ exposure fields in 2014-2017. Environ. Sci. Technol. 53, 7306-7315 (2019).

26. Census Data (National Bureau of Statistics of the People's Republic of China, 2011); http://www.stats.gov.cn/tjsj/pcsj/

27. Baidu Migration (Baidu Inc, 2020); https://qianxi.baidu.com/2020/

28. Huang, X.et al. Enhanced secondary pollution offset reduction of primary emissions during COVID-19 lockdown in China. Natl Sci. Rev. https://doi. org/10.1093/nsr/nwaa137 (2020).

29. Qi, M. et al. Exposure and health impact evaluation based on simultaneous measurement of indoor and ambient $\mathrm{PM}_{2.5}$ in Haidian, Beijing. Environ. Pollut. 220, 704-712 (2017).

30. Notice Regarding the Issuance of Clean Winter Heating Plan in Northern China (2017-2021) (Central People's Government of the People's Republic of China, 5 December 2017); http://www.gov.cn/xinwen/2017-12/20/content 5248855.htm

31. Clean Heating Rate Reached 55\% in 2019 (National Energy Administration, 25 December 2019); https://www.sohu.com/a/362593452_465972

32. Cao, S. et al. Protective behavior of Chinese population wearing masks during the COVID-19 epidemic (in Chinese). Res. Environ. Sci. 33, 1649-1658 (2020)

33. Shen, H. et al. Individual and population level protection from particulate matter exposure by wearing facemasks. Environ. Int. 146, 106026 (2021).

34. Energy Statistics Division of National Bureau of Statistics. China Energy Statistical Yearbook 2018 (China Statistics Press, 2018).

35. Shen, $\mathrm{H}$. et al. Urbanization-induced population migration has reduced ambient $\mathrm{PM}_{25}$ concentrations in China. Sci. Adv. 3, el700300 (2017).

36. Shen, H. et al. Impacts of rural worker migration on ambient air quality and health in China: from the perspective of upgrading residential energy consumption. Environ. Int. 113, 290-299 (2018).

37. Ministry of Environmental Protection. Exposure Factors Handbook of Chinese Population (Adult Volume) (China Environmental Science Press, 2013).

38. Jiang, N. et al. Transportation activity patterns of Chinese population during the COVID-19 epidemic (in Chinese). Res. Environ. Sci. 33, 1675-1682 (2020)

39. AiMa Air Quality Forecasting System (AiMa Forecasts, 6 April 2020); http:// www.aimayubao.com/wryb_eval.php? movie=no

40. Lyu, B., Zhang, Y. \& Hu, Y. Improving $\mathrm{PM}_{2.5}$ air quality model forecasts in China using a bias-correction framework. Atmosphere 8, 147 (2017).

41. CMAQ v.5.0.2 (US EPA Office of Research and Development, 2014); https:// doi.org/10.5281/zenodo. 1079898

42. Skamarock W. C. et al. A Description of the Advanced Research WRF Version 3 (National Center For Atmospheric Research, 2008).

43. NCEP Products Inventory: Global Products, Global Forecast System (GFS) Model (National Centers for Environmental Prediction, 6 April 2020); https:// www.nco.ncep.noaa.gov/pmb/products/gfs/\#GFS 
44. Wade, K. S. et al. Effects of instrument precision and spatial variability on the assessment of the temporal variation of ambient air pollution in Atlanta Georgia. J. Air Waste Manag. Assoc. 56, 876-888 (2006).

45. Goldman, G. T. et al. Ambient air pollutant measurement error: characterization and impacts in a time-series epidemiologic study in Atlanta. Environ. Sci. Technol. 44, 7692-7698 (2010).

46. Zhao, S. et al. A Multiphase CMAQ Version 5.0 Adjoint. Geosci. Model Dev. 13, 2925-2944 (2020).

47. Hakami, A. et al. The adjoint of CMAQ. Environ. Sci. Technol. 41, 7807-7817 (2007).

48. Chen, Y. et al. Transition of household cookfuels in China from 2010 to 2012 Appl. Energy 15, 800-809 (2016).

49. Johnson, M., Lam, N., Brant, S., Gray, C. \& Pennise, D. Modeling indoor air pollution from cookstove emissions in developing countries using a Monte Carlo single-box model. Atmos. Environ. 45, 3237-3243 (2011).

50. WHO Guidelines for Indoor Air Quality: Household Fuel Combustion - Review 3: Model for Linking Household Energy Use with Indoor Air Quality (World Health Organization, 2014).

51. Xiang, J. B. et al. Reducing indoor levels of "outdoor $\mathrm{PM}_{2.5}$ " in urban China: impact on mortalities. Environ. Sci. Technol. 53, 3119-3127 (2019).

52. Kirkwood, T. B. Geometric means and measures of dispersion. Biometrics 35 908-909 (1979).

53. Endo, Y. Estimate of confidence intervals for geometric mean diameter and geometric standard deviation of lognormal size distribution. Powder Technol. 193, 154-161 (2009).

54. Shen, H. et al. Global atmospheric emissions of polycyclic aromatic hydrocarbons from 1960 to 2008 and future predictions. Environ. Sci. Technol. 47, 6415-6424 (2013).

55. Shi, S., Chen, C. \& Zhao, B. Modifications of exposure to ambient particulate matter: tackling bias in using ambient concentration as surrogate with particle infiltration factor and ambient exposure factor. Environ. Pollut. 220, 337-347 (2017).

56. Chen, Y., Ebenstein, A., Greenstone, M. \& Li, H. Evidence on the impact of sustained exposure to air pollution on life expectancy from China's Huai River policy. Proc. Natl Acad. Sci. USA 110, 12936-12941 (2013).

57. Digital Map Database of China, Provincial Boundary (Harvard Dataverse V1, 2020); https://doi.org/10.7910/DVN/DBJ3BX

\section{Acknowledgements}

This paper is dedicated to the memory of Kirk R. Smith, who passed away on 15 June 2020. We thank H. Xu, W. Zhang, X. Yu, Y. Ren and W. Hou for their help with the energy data collection and the indoor model development. This work is funded by the National Natural Science Foundation of China (grant nos. 41991312, 41922057, 41830641 and 41821005), the Chinese Academy of Science (grant no. XDA23010100), the US Environmental Protection Agency (EPA grant no. R835880) and the National Science Foundation (NSF SRN grant no. 1444745). The funders had no role in study design, data collection and analysis, decision to publish or preparation of the manuscript. Its contents are solely the responsibility of the grantee and do not necessarily represent the official views of the supporting agencies. Further, the US government does not endorse the purchase of any commercial products or services mentioned in the publication.

\section{Author contributions}

K.R.S. conceptualized the study. H.S., G.S., Y.C. and S.T. contributed to the study design. H.S. conducted the research and performed analyses with contributions from all authors as specified below. A.G.R. supervised outdoor air quality modelling. Y.H. and B.L. provided the fused $\mathrm{PM}_{2.5}$ field. X.D. provided the time-activity data. W.M., G.S. and H.S. prepared the energy consumption data. Y.X. and H.S. downloaded and prepared the Baidu mobility data. Y.C., W.M. and X.Y. assisted with indoor exposure assessment. S.Z. and A.H. provided the CMAQ-ADJ source code. J.G. provided $\mathrm{PM}_{2.5}$ observation data from the China Meteorological Administration network. H.S. wrote the initial manuscript and provided visualization. K.R.S., H.S., G.S. and Y.C. reviewed the manuscript. All authors edited the manuscript.

\section{Competing interests}

The authors declare no competing interests.

\section{Additional information}

Supplementary information is available for this paper at https://doi.org/10.1038/ s41562-020-01018-Z.

Correspondence and requests for materials should be addressed to G.S.

Peer review information Nature Human Behaviour thanks Patrick Kinney, Yang Liu and Qizhong Wu for their contribution to the peer review of this work. Peer reviewer reports are available. Primary Handling Editor: Aisha Bradshaw.

Reprints and permissions information is available at www.nature.com/reprints.

Publisher's note Springer Nature remains neutral with regard to jurisdictional claims in published maps and institutional affiliations.

(c) The Author(s), under exclusive licence to Springer Nature Limited 2020 


\section{Reporting Summary}

Nature Research wishes to improve the reproducibility of the work that we publish. This form provides structure for consistency and transparency in reporting. For further information on Nature Research policies, see our Editorial Policies and the Editorial Policy Checklist.

\section{Statistics}

For all statistical analyses, confirm that the following items are present in the figure legend, table legend, main text, or Methods section.

n/a Confirmed

$\square \bigotimes$ The exact sample size $(n)$ for each experimental group/condition, given as a discrete number and unit of measurement

Х $\square$ A statement on whether measurements were taken from distinct samples or whether the same sample was measured repeatedly

$\triangle$ The statistical test(s) used AND whether they are one- or two-sided

X Only common tests should be described solely by name; describe more complex techniques in the Methods section.

Х $\square$ A description of all covariates tested

Х $\square$ A description of any assumptions or corrections, such as tests of normality and adjustment for multiple comparisons

$\searrow$ A full description of the statistical parameters including central tendency (e.g. means) or other basic estimates (e.g. regression coefficient)

$\bigotimes$ AND variation (e.g. standard deviation) or associated estimates of uncertainty (e.g. confidence intervals)

X For null hypothesis testing, the test statistic (e.g. $F, t, r$ ) with confidence intervals, effect sizes, degrees of freedom and $P$ value noted

Give $P$ values as exact values whenever suitable.

\ $\square$ For Bayesian analysis, information on the choice of priors and Markov chain Monte Carlo settings

$\bigotimes \square$ For hierarchical and complex designs, identification of the appropriate level for tests and full reporting of outcomes

$\bigotimes \square$ Estimates of effect sizes (e.g. Cohen's $d$, Pearson's $r$ ), indicating how they were calculated

Our web collection on statistics for biologists contains articles on many of the points above.

\section{Software and code}

Policy information about availability of computer code

Data collection No software was used to collect the data.

Data analysis MATLAB R2019b was used to analyze the data.

For manuscripts utilizing custom algorithms or software that are central to the research but not yet described in published literature, software must be made available to editors and reviewers. We strongly encourage code deposition in a community repository (e.g. GitHub). See the Nature Research guidelines for submitting code \& software for further information.

\section{Data}

Policy information about availability of data

All manuscripts must include a data availability statement. This statement should provide the following information, where applicable:

- Accession codes, unique identifiers, or web links for publicly available datasets

- A list of figures that have associated raw data

- A description of any restrictions on data availability

The population distribution data, the daily cross-province migration data, the daily ground-level PM2.5 fusion data, going-out frequencies by gender and age during the COVID-19 quarantine, and all data used to generate the figures in the main text are openly available on Open Science Framework at https://osf.io/x46tb/. The provincial-level time activity data used in this study are provided in Supplementary Table 1 . Further information and requests should be directed to and will be fulfilled by the corresponding author (gfshen12@pku.edu.cn). 


\section{Field-specific reporting}

Please select the one below that is the best fit for your research. If you are not sure, read the appropriate sections before making your selection.

Life sciences $\quad \square$ Behavioural \& social sciences $\square$ Ecological, evolutionary \& environmental sciences

For a reference copy of the document with all sections, see nature.com/documents/nr-reporting-summary-flat.pdf

\section{Behavioural \& social sciences study design}

All studies must disclose on these points even when the disclosure is negative.

Study description

This study evaluates the impacts of the responses to the COVID-19 outbreak on population exposure to PM2.5 in China. The exposure assessment considers both indoor and outdoor PM2.5 exposure and integrates human mobility data and questionnaire survey data with air quality and indoor exposure models.

Research sample The information on the frequencies of going out during the COVID-19 quarantine were obtained from an on-line questionnaire survey (https://www.wjx.cn/m/59666734.aspx). This survey adopted strict quality control measures during data processing and analysis. The questionnaires with missing values, logical errors and data format errors were excluded. 8330 questionnaires were distributed with a recovery rate of $100 \%$. Among them, there were 7784 valid questionnaires, and the response rate was $93.4 \%$. The valid questionnaires covered 31 provinces in China.

The national census data recording the cross-province migration information were obtained at http://www.stats.gov.cn/tjsj/pcsj/ rkpc/6rp/indexch.htm.

Mobility data were freely downloaded from the Baidu real-time mobility monitoring platform (https://qianxi.baidu.com/2020/). Indoor measurements were conducted in 30 households in a village in Jinan, Shandong province. This is a part of an ongoing field monitoring campaign aiming at evaluating indoor air pollution in rural Chinese households.

Sampling strategy For the online questionnaire survey, sample size was determined by the total number of participants filling up the form. For the indoor measurements, as stated in the caption of Supplementary Figure $\$ 8$, the sample size was limited by our sampling capacity and may not be sufficient for representing regional average.

Data collection

The information on the frequencies of going out during the COVID-19 quarantine were collected via an on-line questionnaire survey. The national census data were directly obtained from an online repository (http://www.stats.gov.cn/tjsj/pcsj/rkpc/6rp/indexch.htm). The baseline population distribution data were derived based on satellite nighttime light observation and national census following Shen et al (2017). The dynamic population distributions during the study period were derived by integrating mobility information with baseline population distribution and are freely available at https://osf.io/x46tb/. The ambient PM2.5 concentrations were obtained by combining hourly ground-level observations reported by the China National Urban Air Quality Real-time Publishing Platform with model predictions by the Community Multiscale Air Quality (CMAQ) model version 5.0.2 using an ensemble deep learning data fusion approach following Lyu et al (2019).

The mobility data were directly obtained from the Baidu moility monitoring platform (https://qianxi.baidu.com/2020/) Indoor PM2.5 concentrations were monitored by pre-calibrated sensors following the method described by Lu et al (2020).

Reference:

Lu, C. et al. A novel model for regional indoor PM2.5 quantification with both external and internal contributions included. Environ. Int. 145, 106124 (2020).

Shen, H. et al. Urbanization-induced population migration has reduced ambient PM2.5 concentrations in China. Sci. Adv. 3, e1700300 (2017).

Lyu, B. et al. Fusion method combining ground-level observations with chemical transport model predictions using an ensemble deep learning framework: application in China to estimate spatiotemporally-resolved PM2.5 exposure fields in 2014-2017. Environ. Sci. Technol. 53, 7306-7315 (2019).

Timing

The on-line questionnaire survey started after the outbreak of COVID-19 until March 16, 2020 when the new COVID-19 cases in China were low and people gradually resumed daily life. The survey covered the 31 provinces in China. The Baidu mobility data spanned from January 1, 2020 to March 25, 2020.

The indoor measurements were conducted in a village in Jinan, Shandong province. The measurements were started from January 14th, 2020 and are still in operation.

Data exclusions $\quad$ The questionnaires with missing values, logical errors and data format errors were excluded. No data in the national census, the Baidu mobility, or the indoor measurements were excluded.

Non-participation No participants dropped out/declined participation

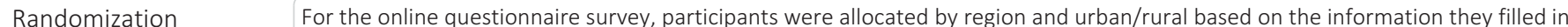
For the indoor measurements, households under investigation were randomly determined.

\section{Reporting for specific materials, systems and methods}

We require information from authors about some types of materials, experimental systems and methods used in many studies. Here, indicate whether each material, system or method listed is relevant to your study. If you are not sure if a list item applies to your research, read the appropriate section before selecting a response. 
Materials \& experimental systems Methods

\begin{tabular}{l|l|l|l|l}
\hline Involved in the study & Antibodies \\
$\square$ Eukaryotic cell lines
\end{tabular}

